\title{
The Efficacy and Safety of Utilizing Mobile Phone- Based Programs and Motion-Capture Devices for Telerehabilitation After Arthroscopic Repair of Rotator Cuff Tear: Study Protocol for a Randomized Controlled Trial
}

\section{Yuanhao Zhang}

Sun Yat-sen memorial hospital Sun Yat-sen University

Jingyi Hou

Sun Yat-sen Memorial Hospital Sun Yat-sen University

\section{Fangqi Li}

Sun Yat-sen Memorial Hospital Sun Yat-sen University

\section{Congda Zhang}

Sun Yat-sen Memorial Hospital Sun Yat-sen University

\section{Yaping Yang}

Sun Yat-sen Memorial Hospital Sun Yat-sen University

Yi Long

Sun Yat-sen Memorial Hospital Sun Yat-sen University

Yiyong Tang

Sun Yat-sen Memorial Hospital Sun Yat-sen University

Rui Yang ( $\nabla$ yangr@mail.sysu.edu.cn )

Sun Yat-sen Memorial Hospital Sun Yat-sen University

\section{Study protocol}

Keywords: rotator cuff tear, arthroscopy, mobile phone, rehabilitation

Posted Date: November 17th, 2020

DOI: https://doi.org/10.21203/rs.3.rs-36193/v1

License: (1) (i) This work is licensed under a Creative Commons Attribution 4.0 International License. Read Full License 


\section{Abstract}

Background: Rotator cuff tear is one of the most common diseases in orthopedics, which seriously affects the quality of patients' lives. And the arthroscopic repair of rotator cuff has recently become more and more popular. Systematic rehabilitation makes great significance for improving the prognosis of postoperative patients. Yet, the traditional outpatient rehabilitation is hard to popularize in developing countries like China due to the limitation of traffic and geography. Given this, we plan to develop a telerehabilitation system to facilitate doctors' remote guidance on patients' rehabilitation.

Methods/design: Our study is a single-center, prospective randomized controlled trial. 124 patients who underwent arthroscopic rotator cuff repair will be recruited for the study. They will be randomly divided into 2 groups (62 cases in each group) based on the stratification factors of the operator, operation and preoperative diagnosis. The patients in the control group will get clinic and booklet based rehabilitation treatment after operation. However, patients in the experimental group will receive mobile phone and motion-capture device based programs for telerehabilitation after surgery. The primary outcome will be measured by the American Shoulder and Elbow Surgeons (ASES). Secondary outcomes include the Range of motion (ROM), Visual Analogue Scales (VAS), EuroQol-5 Dimension health questionnaire (EQ$5 \mathrm{D})$, University of California at Los Angeles $囚 \mathrm{UCLA}$ \and the retear rate.

Discussion: We hypothesize that patients who utilized mobile phone and motion-capture device based telerehabilitation will benefit more in the range of motion and shoulder function than those who received outpatient and manual based rehabilitation. If the hypothesize was confirmed, we could facilitate telerehabilitation for doctors and overcome the geographical and traffic limitations of traditional clinical based rehabilitation.

Trial registration: ChiCTR.org.cn, ChiCTR2000030150, Registered on 24 February 2020

\section{Background}

Rotator cuff tear is a common disease of the shoulder joint with symptoms as shoulder pain, tenderness, limited range of motion and muscle atrophy. It is one of the main causes for shoulder pain and dysfunction and seriously affects the quality of patients' lives. Its incidence rate accounts for $17 \% \sim 41 \%$ of shoulder joint diseases, and increases with age[1]. In the United States, the incidence is $2.1 \%$ for people aged 50 to $60,5.7 \%$ for people aged 60 to 70 , and $30 \%$ for people aged 70 to $80[2]$.

With the progress of technique, arthroscopic rotator cuff repair is more and more widely applied. Over the traditional procedures, we found that it has great advantages for the arthroscopic repair in the excellent visualization, accurately assessing the lesions and minimally invasive procedure. It also provides a tendon-bone interface better suited for biologic healing and restoring normal anatomy[3, 4]. However, the regenerate ability of the damaged tendon is very limited. And it generally takes 6-8 weeks for tendonbone healing, during which the shoulder joint is routinely placed in the abduction resting position to 
reduce the tension of the sutures after operation[5-7]. Therefore, postoperative patients have to face the challenges of recovering the muscle strength and mobility of injured shoulders.

Proper rehabilitation treatment plays an important role in the recovery of shoulder joint function after operation. Many studies have confirmed that the systemic functional exercise of shoulder joint after surgery is helpful to restore the normal functions of the shoulder joint. At the same time, exercise can prevent them from mobility limitation by stretching the shoulder. It can also promote the secretion and circulation of synovial fluid in the joint to reduce intra-articular adhesion[8, 9]. At present, many studies have confirmed that early postoperative rehabilitation is more beneficial to the recovery of patients than delayed rehabilitation[10,11]. Other studies reported the effect of rehabilitation on ROM: Lee et al. compared the different intensive systematic rehabilitation which were started 6 weeks after operation and found that patients with higher intensity rehabilitation programs had larger ROM 3 months after operation[9]. Cuff and Pupello et al. found that the shoulder joint flexion of patients who received rehabilitation earlier after operation reached $172^{\circ} 6$ months after operation, while that of patients who received delayed rehabilitation only reached $165^{\circ}[12]$. These studies show that early rehabilitation programs have a great impact on the prognosis of patients. However, in clinical practice, we found that the traditional rehabilitation plan is hard to apply to all patients.

First of all, the current rehabilitation is mainly carried out by attending outpatient rehabilitation courses. Based on the biological characteristics of rotator cuff tear recovery process, the whole postoperative rehabilitation plan for rotator cuff tear can be divided into three stages: stage one (0-6w after surgery) is mainly aimed at preventing disused muscular atrophy and joint adhesion. And the rehabilitation focus on passive activity. Stage two (7-12w after operation) concentrates on the normal motion recovery through the active movement. Moreover, muscle strength training can be started. Stage three (after $12 \mathrm{w}$ postoperation) focuses on restoring the neuromuscular control ability around the shoulder joint and further enhancing the muscle strength training[12,13]. At the beginning of each stage, patients need to return to hospital for guidance. If patients want to complete the whole systematic rehabilitation, they need to return to hospital at least 3-12 times with the time span as long as 12 weeks, leading to the decrease in patient compliance.

Secondly, rehabilitation is a long-term process that requires continuous guidance and encouragement to patients. In the postoperative rehabilitation of rotator cuff tear, in order to gain the best prognosis, stretching and strength exercise should continue until 1 year after operation[14]. To a large extent, this depends on the patients' exercise at home especially in the late period. However, in the traditional clinic rehabilitation mode, it is difficult for doctors to accurately supervise the process of patients' rehabilitation. And there is also a deficiency of objective indicators for the evaluation of patients' compliance[15]. Moreover, due to the lack of necessary communication methods, the patient's compliance in the late period of exercise decreases and the long-term prognosis becomes worse.

In addition, due to maldistribution of medical resources, many patients have to travel a long distance to get their operations. Therefore, it is particularly necessary to find a convenient telerehabilitation platform 
that can provide patients with guidance and communication of doctors.

We made an attempt on this issue in 2012. As a result, we designed a telerehabilitation platform based on mobile phones. Through this platform, we can make and modify doctors' rehabilitation plans for patients from time to time, and guide patients in the form of videos to carry out rehabilitation. Also, a clinical experiment based on this platform was conducted and confirmed its safety and effectiveness, which has already been published[16].

Meanwhile during the preliminary test, we found that the patients' compliance decreased because they could not know whether their rehabilitation actions were standard. Given this, we developed a real-time motion monitoring system for postoperative rehabilitation training. Through this system, we could supervise patients' postoperative rehabilitation with high precision in real-time. Thus, we may be able to further improve the accuracy and compliance of patients, which is expected to improve the prognosis of patients.

\section{Methods/design}

This protocol is referred to the SPIRIT checklist (Additional file 1)[17].

\section{Study design}

This is a Single-center, Prospective Randomized Controlled Trial with a two-group parallel design. The patients in the control group will be given clinic and booklet guidance for rehabilitation treatment after operation. However, patients in the experimental group will receive mobile phone and motion-capture device based programs for telerehabilitation after surgery. The patients will be followed up for 1 year ( 3 months, 6 months and 1 year) and completed the relevant evaluations.

\section{Aim}

1. This trial plans to develop a telerehabilitation training platform based on mobile phones and motion monitors for patients with rotator cuff tears to realize the remote guidance of doctors for patients' rehabilitation training, aiming to overcome the geographical and traffic restrictions of traditional clinic based rehabilitation;

2. Through a well-designed randomized controlled study, we aim to verify the effectiveness and safety of mobile phones and motion-capture devices based telerehabilitation system for patients with rotator cuff tears.

The flow chart is as follows (Fig 1)

Fig.1 Study flow chart

\section{Patients and settings}


Patients will be recruited from the Department of Orthropedics, Sun Yat-sen Memorial Hospital, Sun Yatsen University in China.

Patients may be enrolled in the study provided they meet all of the inclusion criteria:

- Aged between 16 and 64 years, owing a mobile phone

- Agreed to receive arthroscopic repair of rotator cuff

- Diagnosed as small to medium full-thickness rotator cuff tear(less than $3 \mathrm{~cm}$ ), with imaging support

- Living at least $100 \mathrm{~km}$ or a 2 hours' drive away from the hospitals $₫ 5 \llbracket$ Signed the informed consent.

Patients are excluded if they fulfill any of the following exclusion criteria:

- Diagnosed as tuberculosis, shoulder joint infections or tumors.

- Those who accepted rotator cuff repair or other major shoulder joint surgeries before this trial.

- Patients with large or massive rotator cuff tear (more than $3 \mathrm{~cm}$ ) or other shoulder joint injury as SLAP injury or Bankart injury.

- Patients with shoulder dysfunction (neurogenic paralysis).

- Those who could not complete the rehabilitation due to mental retardation or other reasons.

\section{Recruitment}

Orthopedic surgeons conducted a preliminary screening of all patients by collecting gender, diagnosis, size and location of rotator cuff tears of the injured limb. Once patients are identified and confirmed to suffer rotator cuff tears and reach the age of 16 , they are transferred to the researchers who are responsible for the qualification program. In general, researchers will provide patients with detailed information about the study when performing a preoperative evaluation or other convenient time before operation. They will seek for the patients' written informed consent. And patients who are interested in and qualified for the project must provide written informed consent before participating. If patients agree to participate in this trial, they will be included in the study when the surgeons in charge have evaluated their rotator cuff as repairable and confirmed by the operation later.

We adopt the recruitment method by introducing the trial to the patients. The recruitment site is the outpatient department of orthopedics or the inpatient department of sports medicine of Sun Yat-Sen Memorial Hospital of Sun Yat-sen University. The whole recruitment process should be non-mandatory. In case of the expenses that can be exempted by the subjects, the recruitment shall be as clear as possible to avoid using such general terms as "free treatment" or "free laboratory test" to avoid misunderstanding. Special attention should be paid to avoid recruitment of the following subjects:

1. Patients who have difficulty in understanding or complying with the program, due to various reasons such as poor understanding, mental disorder or language barrier;

2. Patients who tend to lead to disputes between doctors and patients; 
3. Patients who are participating in other experimental studies.

\section{Data collection}

For every patient to enter the screening, the screened patients will enter the randomization group after signing the informed consent. And their CRF must be completed and signed by the main investigator or the authorized investigator. Researchers should ensure the accuracy and completeness of all data. When the clinical supervisor submits the CRF, the data administrator shall check, count and accept the CRF according to the supervisor's delivery sheet. If the acceptance is confirmed, the CRF delivery sheet shall be signed and properly kept in time. The data administrators use REDCAP network electronic data management system to input data, check the original case report one by one and correct it according to the final CRF. After data entry, the data verifier shall use computer SAS program and manual inspection according to the requirements of CRF verification plan. The content of verification includes data integrity, consistency, logicality and scheme violation and deviation, such as inclusion and exclusion standard inspection, missing value inspection, time window inspection, logical relationship inspection, laboratory inspection and judgment inspection. If there is any question, doctors can fill in the data question form. Researchers will answer the questions in written form and sign them, and then return them to the data administrator. The data administrator shall check item by item and make it correction and confirmation. After the above work is completed, we will close the data. After the data closed, it will be audited. After the database passes the audit and is considered to be correct, the data will be locked by the main researcher, the sponsor, the statistical analyst, the supervision and management personnel. The locked data file cannot be changed. The database will be submitted to the statistical analysts for statistical analysis according to the requirements of the statistical plan.

\section{The randomization scheme}

The subject is randomly divided into two groups with the proportion of 1:1. The statistician will generate random digital code through SAS 9.2 software simulation, list the corresponding rehabilitation scheme allocation and then seal the allocation scheme with opaque envelopes. After the examination, according to the random number generated by the computer and the order in which the subjects are enrolled, the patients will be randomly assigned treatment options. The eligible patients will be randomized to the experimental group (telerehabilitation treatment guided by mobile phone and motion-capture device based programs) and the control group (rehabilitation treatment guided by clinic and booklet) according to the proportion of $1: 1$.

\section{Blinding}

This test is an "open label" trial. First, the orthopedic doctors will carry out preliminary screening; then, the therapists will inform the participants of the distribution, and provide the corresponding treatment of the two groups of patients. The assessments of this study are divided into subjective and objective evolution. Subjective evolution including ASES, VAS and EQ-5D, will be assessed by patients themselves and cannot be blinded, since the patients are aware of their allocation. Objective evolution includes ROM, UCLA and 
retear rate. In the assessment of this part, we will implement the blinding for assessors. Assessors will not be aware of the allocation and the patients will not be allowed to disclose their allocation at the time of assessment at 3 months, 6 months and 1 year after surgery. And the effect of blinding will be tested by asking the assessor to determine the group of patients. However, if (serious) adverse events were happened, blinded person will be informed to participate in the resolution of the event.

\section{Interventions}

Both groups will undergo arthroscopic rotator cuff repair according to the specific diagnosis and condition of the patients. And the operations will be performed according to the routine of the operators. At the same time, the preoperative diagnosis, surgical options and specific operation process of the patients will be recorded in detail. After operation, patients will wear shoulder abduction sling for 6 weeks. And before discharge, the therapist will have a comprehensive communication with patients, including the position and time of wearing the shoulder abduction sling. Generally, we suggest that patients should wear the abduction sling strictly for 6 weeks, and it can only be removed for specific reasons such as dinning etc. At the same time, the therapist will guide patients to perform rehabilitation actions at this stage, including shrugging, chest expansion, wrist and elbow exercises until discharge.

Control group will be treated with routine rehabilitation. We refer to the rehabilitation consensus of the American Shoulder and elbow Association. [18]Generally, before discharge, we will provide a postoperative information and exercise manual, which details the rehabilitation and the precautions that patients need to perform after discharge. That manual includes photos and texts. And it details the exercise type, action combination and number of repetitions. Additionally, it also explains when to return to hospital for rehabilitation guidance after operation. Every time patients return, doctors will give guidance to their rehabilitation and demonstrate the new actions they need to conduct until the patient can smoothly complete the whole set of rehabilitation actions.

Intervention group will receive telerehabilitation treatment. Different from the control group, besides the usual practice of the relevant surgeons, patients who are randomly assigned to participate in the intervention group will also receive mobile phone and motion-capture device based programs independently designed and developed by the experimenter. After the operation, enrolled patients will install the platform. At the same time, researchers will show patients how to use and practice. And they will evaluate patients to ensure that they can correctly perform rehabilitation training through the platform. The rehabilitation plan will last for 1 year. Patients who completed 5 or more training sessions each week will be considered as high adherence, 3 to 5 training sessions as medium adherence, and 2 training sessions and less as low adherence.

The telerehabilitation system is divided into mobile client and web client. The mobile client can be applied to android and IOS system. Through the system, patients can view the rehabilitation training plan made by doctors and perform rehabilitation treatment according to the actions in the rehabilitation video. Rehabilitation training consists of 22 sections, including passive joint activity training, active joint activity training and muscle strength training. Through the system, doctors can arbitrarily arrange the 
combination of sections and the number of exercises for each section. The system will record the progress of patient's rehabilitation and warn patients who have not completed the training. The patient's daily rehabilitation progress can also be fed back to the doctor. Through this system, doctors can view patients' rehabilitation training reports, understand the patient's acceptance of the rehabilitation plan and resolve related issues. At the same time, by cooperating with the motion-capture device used by the above rehabilitation platform, the therapist can obtain the flexion and extension angle, angular velocity, acceleration and other data of the joint in time to realize the real-time detection of the postoperative rehabilitation motion of patients.

The motion-capture device based programs is as follows (Fig 2)

Fig.2 (A) The motion-capture device is based on six axis inertial sensor and it can monitor the patients' range of motion etc. (B) The software interface: the upper part shows the video guidance, and the lower part is the instrument panel to display the range of motion. (C) Patients rehabilitate using the system: The patient can rehabilitate according to the video guidance. Meanwhile the information such as the range of motion is transmitted back to the mobile phone by the motion-capture device they wear, and displayed on the mobile phone, so that the patients can know whether their actions reach the standard.

When patients return to the hospital, the senior therapist will provide health education to patients. They will inform the prognosis of related diseases, the necessity and precautions of rehabilitations. Meanwhile, they will guide and standardize the patient's actions. After that, patients will exercise at home according to the mobile phone and motion-capture device based programs. If patients have any questions, they can directly communicate with the therapist through platform to adjust the rehabilitation plan. All data recorded will be synchronized and stored in the remote server.

All patients need to return to the hospital for evaluation at 3 months, 6 months and 1 year after the operation, and complete the relevant scale.

\section{Study training}

Before the clinical trial, we will finish all the training tasks. At the same time, we will train all the researchers on the trial scheme, including the content of informed consent, the specific rehabilitation training plan after arthroscopy, the application of mobile phone software and motion device, the filling of case report form (CRF), the complete retention of examination records, clinical records and the original medical records of patients; the communication channel with the sponsor, adverse event report, etc. Before recruitment and treatment, the inpatient department and outpatient rehabilitation physiotherapists should be familiar with the content of the clinical trial plan, master the principle of GCP, unify the recording method and judgment standard, and strictly follow the plan. Because our physiotherapists are limited, we will explain to the postoperative patients by holding a meeting.

Our training will be conducted in the form of lectures, including lectures on clinical program explanation, CRF filling, implementation of inclusion and exclusion criteria, adverse event handling, etc. The study 
leader will regularly check the original medical records and medication history of the selected patients, observe the research progress and discuss the treatment with the researchers.

\section{Outcome measures/variables}

The researchers will provide baseline questionnaires for the patients who have been enrolled. First, they will collect the background information including demographic data $\llbracket$ date of birth, gender, height, weight, nationality, marital status, occupation, education level, etc. and general clinical data: course of disease, current history, past history, combined disease, drug use and allergy history. Secondly, the patients will fill in the clinical efficacy indicators. In order to evaluate the effect of telerehabilitation based system, the American Shoulder and Elbow Surgeons (ASES) is evaluated as the primary outcome. Secondary outcome are collected to evaluate the influence of mental health, life status, health economics and longterm prognosis of patients, including range of motion (ROM), visual analog scale of pain Scales (VAS score); EuroQol-5 Dimension health questionnaire (EQ-5D) ; University of California at Los Angeles (UCLA) and retear rate.

We have evaluated patients who had been enrolled at baseline (preoperative); 3,6 , and 12 months after baseline.(Fig 3)

Fig.3 Time points for enrollment, interventions and assessments

The primary outcome is the American Shoulder and Elbow Surgeons (ASES). It is a survey and evaluation in the form of interviewing patients with highly effectiveness. Meanwhile, it has three characteristics: 1. Easy to use 2. The method of evaluating Activities of Daily Living (ADL) 3. Including the self-evaluation part of patients[19]. The ASES questionnaire consists of three parts: 1. Pain (6 items), 2. Instability ( 2 items), 3. ADL (10 items). Pain and instability are binary answers (yes / no), visual analogue scale (VAS) for pain and instability (where $0=$ best, $10=$ worst), and 4-point sequential scale assessment representing function (where $0=$ unable, 1 = very difficult, $2=$ somewhat difficult, and $3=$ not difficult)[20]. Unstable items and other 5 pain items will not affect the total score of ASES. At the same time, we evaluate the prognosis according to the final score: 0 is the worst, 100 is the best[21].

The University of California at Los Angeles (UCLA) is used to evaluate the function and quality of life of the patients after operation, and it also has good reliability and validity. It mainly includes five areas with different scores: pain (10 points), function (10 points), active flexion (5 points), flexion strength (5 points) and overall satisfaction (5 points). Finally, by adding all the scores, the total score is 35 points. The higher the score implying the better function recovery in the shoulder[22]. EuroQol-5 Dimension health questionnaire (EQ-5D) is a general index to judge the health status of patients. It requires patients to state whether they have no, some or extreme problems in the following five health aspects: activity ability, selfcare, daily activities, pain / discomfort and anxiety / depression. Generally, the scale uses five sequential options (no problem, minor problem, moderate problem, serious problem and extreme problem) to indicate the severity of the problem[23]. We use this questionnaire to assess the health-related quality of life of the enrolled patients. 
In addition, ROM, VAS and retear rate are added to our secondary indexes to evaluate the prognosis of patients.

\section{Sample size calculation}

This is a single center prospective randomized controlled trial, so it is necessary to ensure that there is enough sample size to detect the difference between outpatient and manual guidance rehabilitation treatment and telerehabilitation of mobile phones and motion detectors. After calculation, 124 patients will be included in the trial at least. And they will be randomly divided into two groups (62 in each group) according to the ratio of 1:1. The basis of sample size calculation is as follows: This study is a noninferiority test, with American Shoulder and Elbow Surgeons (ASES) as the primary outcome. Based on the results of previous studies, we estimate that the final mean of ASES in the control group is 88.1 (SD 15.8)[24]. We assume that the standard deviation of ASES in the experimental group is 14.1, and the noninferiority bound $(\delta)$ is 1.5 . Type I error: two sided $a=0.025$ test validity $90 \%$. At the same time, according to the loss rate of $10 \%$ to calculate, we need to recruit at least 62 people in each group.

\section{Planned recruitment rate}

The planned recruitment rate is ten or eleven participants per month within 12 months (starting in March, 2020), reaching 124 recruitment targets.

\section{Safety monitoring}

The number of adverse events predicted in this experiment is minimal, and the intervention measures are not high-risk. Therefore, the data monitoring committee is composed of members of the quality control department of this subject, who are responsible for the data and safety evaluation. In the event of an adverse event, members of the data monitoring committee will assess whether the number of adverse events has reached the threshold that needs to be stopped early. In addition, if the patient has extreme deterioration of symptoms during rehabilitation treatment and these symptoms persist, the therapist will report them to the committee members and evaluate them through orthopedic experts. All adverse events will be recorded in the CRF.

\section{Ethics}

This study will collect the clinical data, personal information for scientific research, which will involve the privacy rights and interests of patients. Participants and data analysts of this study will sign a confidentiality agreement and will not disclose personal information and disease-related information of patients to any individuals and institutions unrelated to this study. The collected patient data shall be managed uniformly to prevent personal privacy leakage.

Before screening, researchers will verbally inform all potential patients of the purpose, process, potential risks, costs and benefits of this trial. A written informed consent must be signed before the patient participating in the trial. At the same time, all patients have the right to withdraw from the trial at any time 
without affecting their treatment at any time or place. If the patient decides to withdraw from the trial, the researcher shall know the reason as much as possible and record the reason on the case report form. Meanwhile, the researcher shall contact the patient as much as possible, record the last treatment time, and complete the evaluation items that can be completed. If patients withdraw from the trial due to adverse drug events / serious adverse events (AE / SAE), abnormal test values, these must also be recorded on the case report form. This clinical trial must comply with the declaration of Helsinki (2000 version)[25], the code for clinical trial management of drugs (GCP) issued by CFDA and relevant regulations. Before the experiment, the study can be started only after the scheme is approved by the ethics committee of the leader unit. Any modification of the protocol during the clinical study should be reported to the ethics committee and filed. The study was approved by Sun Yat-Sen Memorial Hospital, hospital ethics committees on December 10, 2019. At present, the study has been registered on ChiCTR.org.cn (ChiCTR2000030150). We intend to share the data for future research after the researcher has released the data set completely.

\section{Statistical analysis plan}

This is a randomized controlled trial (RCT). All statistical analysis will be completed with SAS version 9.2 or later. We will divide the population and calculate the full analysis set, Per protocol set and the safety set. We will carry out inclusion and detachment analysis, demographic data and baseline analysis, compliance analysis of intervention measures, Curative effect analysis (full analysis set and per protocol set) and safety analysis (safety analysis set). Then we will evaluate the mean value and confidence interval of clinical results to determine the changes of indicators. Except for special instructions, all statistical tests adopt the double-sided test with $a=0.05$, and the confidence interval is estimated with the double-sided 95\% confidence interval. Quantitative indicators are described by case number, mean, standard deviation, median, upper and lower quartiles, minimum value and maximum value. Additionally, classification indicators are described by case number and percentage. $T$ test, $\chi 2$ test, Fisher exact probability method and rank sum test are used to compare the baseline equilibrium of the two groups. Research statisticians will formulate detailed statistical analysis plans and obtain the approval of the experimental steering committee. At the end of the study, we will discuss the results of the study with the members of the committee and make suggestions on the experiments conducted.

\section{Discussion}

The evidence to date shows that early rehabilitation activities have a very important impact on the prognosis of patients. Therefore, how to make patients benefit most from early rehabilitation activities is a significant topic. Due to the uneven medical resources in different regions, not all patients will receive strictly designed postoperative rehabilitation. At the same time, the patient's compliance is also an important issue leading to the decline of rehabilitation efficacy. Makhni, EC have studied the compliance of rehabilitation training after shoulder arthroscopy. They found that the patient's compliance before operation was the highest: $76 \%$. As the time after operation was prolonged, the compliance gradually decreased, reaching only $45 \%$ at 12 months after operation. Meanwhile, they found that some patients 
did not fill in the electronic form near the time point of 12 months after operation. This shows that as patients lived farther away from the operation, their interest in filling in rehabilitation information decreases[25]. In addition, in Ahmad S's study, they found that the patient's postoperative compliance is a significant prognostic factor for re-tearing[26]. The above evidences show that the decrease of patients' postoperative compliance will lead to the decrease of clinical efficacy, and also suggest that the problem of long-term compliance decrease of patients with rotator cuff tear cannot be ignored[10].

And the wide application of mobile phones is the basis of our research. In fact, Dejan and others have already explored the application of short message of mobile phone in medical treatment in 2011. Compared with traditional phones, in addition to phone messages, mobile phones can be used for video, animation, network and other means to further promote communication between doctors and patients. To date, there have been eighteen studies reported the application of Internet and smart phones in type II diabetes. It has been confirmed that mobile phone application has become a new trend for type II diabetes patients to self-management[27-44]. The research by Glasgow, Kim et al. suggested that patients with software support were better than the control group[29, 30]. Quinn et al. found that the glycosylated hemoglobin level of patients using smart phone software was $1.2 \%$ lower than that of the control group after 1 year[45]. Therefore, we believe that mobile phones will play a positive role in the rehabilitation treatment after rotator cuff tear. Through the remote guidance of mobile phone and motioncapture device based programs, we can follow up the daily rehabilitation of patients, make a more accurate assessment of the training outcome of patients during the whole process and formulate a more individualized rehabilitation plan to effectively improve the prognosis of patients and overcome the defects of traditional clinical and booklet guidance.

\section{Limitation}

1. This study is the first in China to explore the telerehabilitation treatment of patients after rotator cuff surgery, so the efficacy and safety of the system are uncertain and need to be explored;

2. The patients involved in this study need to receive arthroscopic rotator cuff repair, so the surgical technique itself has a certain impact on the results of this study;

3. Movement monitoring is a cutting-edge multidisciplined technique. The successful development of the rehabilitation platform requires multi-disciplinary technology of sports medicine, rehabilitation medicine, software programming and hardware development, which is a challenge to us.

\section{Trial status}

At the time of submission of this protocol document, this trial (version 1.0 of the agreement of February 24,2020 ) has started recruitment (recruitment started on March 1, 2020). Recruitment is planned to be completed on February 28, 2021.

\section{Declarations}


We would like to thank all the orthopedic experts and members of the research group who participate in the operation. At the same time, we would like to thank the therapists who guide the rehabilitation of the patients and the participating departments of Sun Yat-Sen Memorial Hospital of Sun Yat-sen University. They have made an important contribution on the establishment of this study.

\section{Funding}

Funding was received from Guangzhou Science and Technology Program Key projects(no.201704020132), Sun Yat-Sen Clinical Research Cultivating Program『SYS-Q-202006『and Guangdong Natural Science Foundation (No. 2018A030313780). The funding body did not take part in the design of the study or in the writing of the manuscript.

\section{Availability of data and materials}

We have established a data sharing arrangement, and the data of the clinical trial are all entered into the epidata system. The data custodian and members will review our application for anonymized data access to our research database. Any member who needs to access the database must go through an ethical review. After the completion of the test, the original data will be released.

\section{Dissemination policy}

The results of the study will be published in peer-reviewed journal and informed to the clinicians and participants involved in the study.

\section{Authors'contributions}

JYH designed and initiated the trial. Meanwhile, he applied for funding. FQL, YHZ and CDZ were responsible for logistics, patient recruitment and data collection. RY was responsible for the operating and leading. JYH, YPY and YL carried out experimental design, statistical analysis plan and writing of the manuscript. YHZ drafted the manuscript. And all the authors had read and approved the final manuscript.

\section{Ethics approval and consent to participate}

The study was approved by Sun Yat-Sen Memorial Hospital, hospital ethics committees on December 10, 2019. At present, the study has been registered on chictr.org.cn (ChiCTR2000030150). Researchers must inform patients of the purpose, benefits and potential risks of the trial and obtain the written consent of patients before entering the group.

\section{Consent for publication}

Not applicable

\section{Study sponsor}

This study is sponsored by Sun Yat-Sen Memorial Hospital of Sun Yat-sen University. 


\section{Competing interests}

The authors states that they have no conflict of interests.

\section{References}

1. Lo, I.K. and S.S. Burkhart, Current concepts in arthroscopic rotator cuff repair. Am J Sports Med, 2003. 31(2): p. 308-24.

2. Diebold, G., et al., Relationship Between Age and Rotator Cuff Retear: A Study of 1,600 Consecutive Rotator Cuff Repairs. J Bone Joint Surg Am, 2017. 99(14): p. 1198-1205.

3. Sugaya, H., et al., Functional and structural outcome after arthroscopic full-thickness rotator cuff repair: single-row versus dual-row fixation. Arthroscopy, 2005. 21(11): p. 1307-16.

4. Trung, D.T., et al., Evaluate the Results at Minimum 2-Years of Treating Rotator Cuff Tear by Arthroscopic Surgery. Open Access Maced J Med Sci, 2019. 7(24): p. 4199-4203.

5. Depres-Tremblay, G., et al., Rotator cuff repair: a review of surgical techniques, animal models, and new technologies under development. J Shoulder Elbow Surg, 2016. 25(12): p. 2078-2085.

6. Cottrell, J.A., et al., The Biology of Bone and Ligament Healing. Foot Ankle Clin, 2016. 21(4): p. 739761.

7. Meier, S.W. and J.D. Meier, Rotator cuff repair: the effect of double-row fixation on three-dimensional repair site. J Shoulder Elbow Surg, 2006. 15(6): p. 691-6.

8. Millett, P.J., et al., Rehabilitation of the rotator cuff: an evaluation-based approach. J Am Acad Orthop Surg, 2006. 14(11): p. 599-609.

9. Lee, B.G., N.S. Cho and Y.G. Rhee, Effect of two rehabilitation protocols on range of motion and healing rates after arthroscopic rotator cuff repair: aggressive versus limited early passive exercises. Arthroscopy, 2012. 28(1): p. 34-42.

10. Duzgun, I., G. Baltaci and O.A. Atay, Comparison of slow and accelerated rehabilitation protocol after arthroscopic rotator cuff repair: pain and functional activity. Acta Orthop Traumatol Turc, 2011. 45(1): p. 23-33.

11. Weber SC, T.D., Abstract: Early versus delayed rehabilitation in arthroscopic rotator cuff surgery: A dual surgeon comparative cohort study (SS-15). Arthroscopy 2012;28(6, suppl 1):e9-e10. 2012.

12. Cuff, D.J. and D.R. Pupello, Prospective randomized study of arthroscopic rotator cuff repair using an early versus delayed postoperative physical therapy protocol. J Shoulder Elbow Surg, 2012. 21(11): p. $1450-5$.

13. Ross, D., et al., Rehabilitation following arthroscopic rotator cuff repair: a review of current literature. J Am Acad Orthop Surg, 2014. 22(1): p. 1-9.

14. Hughes, A., et al., Pattern and time phase of shoulder function and power recovery after arthroscopic rotator cuff repair. J Shoulder Elbow Surg, 2012. 21(10): p. 1299-303. 
15. Makhni, E.C., et al., Patient Compliance With Electronic Patient Reported Outcomes Following Shoulder Arthroscopy. Arthroscopy, 2017. 33(11): p. 1940-1946.

16. Hou, J., et al., The Effectiveness and Safety of Utilizing Mobile Phone-Based Programs for Rehabilitation After Lumbar Spinal Surgery: Multicenter, Prospective Randomized Controlled Trial. JMIR Mhealth Uhealth, 2019. 7(2): p. e10201.

17. Chan, A.W., et al., SPIRIT 2013 statement: defining standard protocol items for clinical trials. Ann Intern Med, 2013. 158(3): p. 200-7.

18. Thigpen, C.A., et al., The American Society of Shoulder and Elbow Therapists' consensus statement on rehabilitation following arthroscopic rotator cuff repair. J Shoulder Elbow Surg, 2016. 25(4): p. 521-35.

19. Angst, F., et al., Measures of adult shoulder function: Disabilities of the Arm, Shoulder, and Hand Questionnaire (DASH) and its short version (QuickDASH), Shoulder Pain and Disability Index (SPADI), American Shoulder and Elbow Surgeons (ASES) Society standardized shoulder assessment form, Constant (Murley) Score (CS), Simple Shoulder Test (SST), Oxford Shoulder Score (OSS), Shoulder Disability Questionnaire (SDQ), and Western Ontario Shoulder Instability Index (WOSI). Arthritis Care Res (Hoboken), 2011. 63 Suppl 11: p. S174-88.

20. Richards, R.R., et al., A standardized method for the assessment of shoulder function. J Shoulder Elbow Surg, 1994. 3(6): p. 347-52.

21. Sallay, P.I. and L. Reed, The measurement of normative American Shoulder and Elbow Surgeons scores. J Shoulder Elbow Surg, 2003. 12(6): p. 622-7.

22. Xu, S., et al., Determination of Threshold Scores for Treatment Success After Arthroscopic Rotator Cuff Repair Using Oxford, Constant, and University of California, Los Angeles Shoulder Scores. Arthroscopy, 2019. 35(2): p. 304-311.

23. Devlin, N.J., et al., Valuing health-related quality of life: An EQ-5D-5L value set for England. Health Econ, 2018. 27(1): p. 7-22.

24. Thigpen, C.A., et al., The American Society of Shoulder and Elbow Therapists' consensus statement on rehabilitation following arthroscopic rotator cuff repair. J Shoulder Elbow Surg, 2016. 25(4): p. 521-35.

25. World Medical Association Declaration of Helsinki: ethical principles for medical research involving human subjects. JAMA, 2000. 284(23): p. 3043-5.

26. Ahmad, S., M. Haber and D.J. Bokor, The influence of intraoperative factors and postoperative rehabilitation compliance on the integrity of the rotator cuff after arthroscopic repair. J Shoulder Elbow Surg, 2015. 24(2): p. 229-35.

27. Carter, E.L., G. Nunlee-Bland and C. Callender, A patient-centric, provider-assisted diabetes telehealth self-management intervention for urban minorities. Perspect Health Inf Manag, 2011. 8: p. 1b.

28. Feil, E.G., et al., Who participates in Internet-based self-management programs? A study among novice computer users in a primary care setting. Diabetes Educ, 2000. 26(5): p. 806-11. 
29. Glasgow, R.E., et al., Engagement in a diabetes self-management website: usage patterns and generalizability of program use. J Med Internet Res, 2011. 13(1): p. e9.

30. Kim, C.J. and D.H. Kang, Utility of a Web-based intervention for individuals with type 2 diabetes: the impact on physical activity levels and glycemic control. Comput Inform Nurs, 2006. 24(6): p. 337-45.

31. Liebreich, T., et al., Diabetes NetPLAY: A physical activity website and linked email counselling randomized intervention for individuals with type 2 diabetes. Int J Behav Nutr Phys Act, 2009. 6: p. 18.

32. Lorig, K., et al., Online diabetes self-management program: a randomized study. Diabetes Care, 2010. 33(6): p. 1275-81.

33. Mcllhenny, E.H., et al., Methodology for determining major constituents of ayahuasca and their metabolites in blood. Biomed Chromatogr, 2012. 26(3): p. 301-13.

34. Noh, J.H., et al., Web-based comprehensive information system for self-management of diabetes mellitus. Diabetes Technol Ther, 2010. 12(5): p. 333-7.

35. Richardson, C.R., et al., A randomized trial comparing structured and lifestyle goals in an internetmediated walking program for people with type 2 diabetes. Int J Behav Nutr Phys Act, 2007. 4: p. 59.

36. Dobson, R., et al., Effectiveness of text message based, diabetes self management support programme (SMS4BG): two arm, parallel randomised controlled trial. BMJ, 2018. 361: p. k1959.

37. Al-Ozairi, E., et al., Diabetes and TelecommunicationS (DATES) study to support self-management for people with type 2 diabetes: a randomized controlled trial. BMC Public Health, 2018. 18(1): p. 1249.

38. Rangraz, J.F., et al., Mobile phone usage in patients with type II diabetes and their intention to use it for self-management: a cross-sectional study in Iran. BMC Med Inform Decis Mak, 2020. 20(1): p. 24.

39. Nanditha, A., et al., A pragmatic and scalable strategy using mobile technology to promote sustained lifestyle changes to prevent type 2 diabetes in India and the UK: a randomised controlled trial. Diabetologia, 2020. 63(3): p. 486-496.

40. Huang, Z., et al., A Smartphone App to Improve Medication Adherence in Patients With Type 2 Diabetes in Asia: Feasibility Randomized Controlled Trial. JMIR Mhealth Uhealth, 2019. 7(9): p. e14914.

41. Mayberry, L.S., et al., The Design, Usability, and Feasibility of a Family-Focused Diabetes Self-Care Support mHealth Intervention for Diverse, Low-Income Adults with Type 2 Diabetes. J Diabetes Res, 2016. 2016: p. 7586385.

42. Peimani, M., et al., Effectiveness of short message service-based intervention (SMS) on self-care in type 2 diabetes: A feasibility study. Prim Care Diabetes, 2016. 10(4): p. 251-8.

43. Pal, K., et al., Computer-based interventions to improve self-management in adults with type 2 diabetes: a systematic review and meta-analysis. Diabetes Care, 2014. 37(6): p. 1759-66.

44. Vervloet, M., et al., Short- and long-term effects of real-time medication monitoring with short message service (SMS) reminders for missed doses on the refill adherence of people with Type 2 diabetes: evidence from a randomized controlled trial. Diabet Med, 2014. 31(7): p. 821-8. 
45. Quinn, C.C., et al., Mobile diabetes intervention study: testing a personalized treatment/behavioral communication intervention for blood glucose control. Contemp Clin Trials, 2009. 30(4): p. 334-46.

\section{Figures}

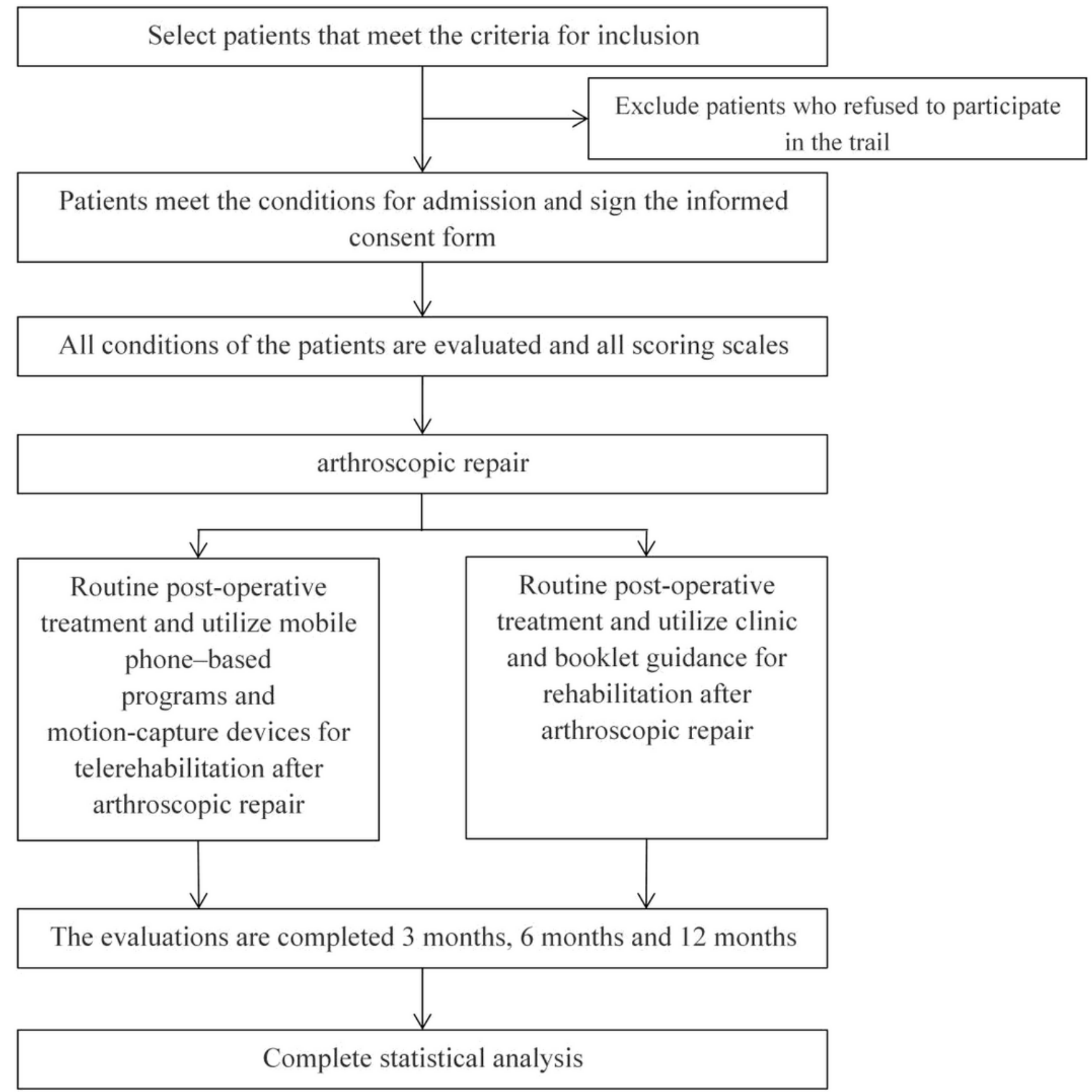

\section{Figure 1}

Study flow chart 

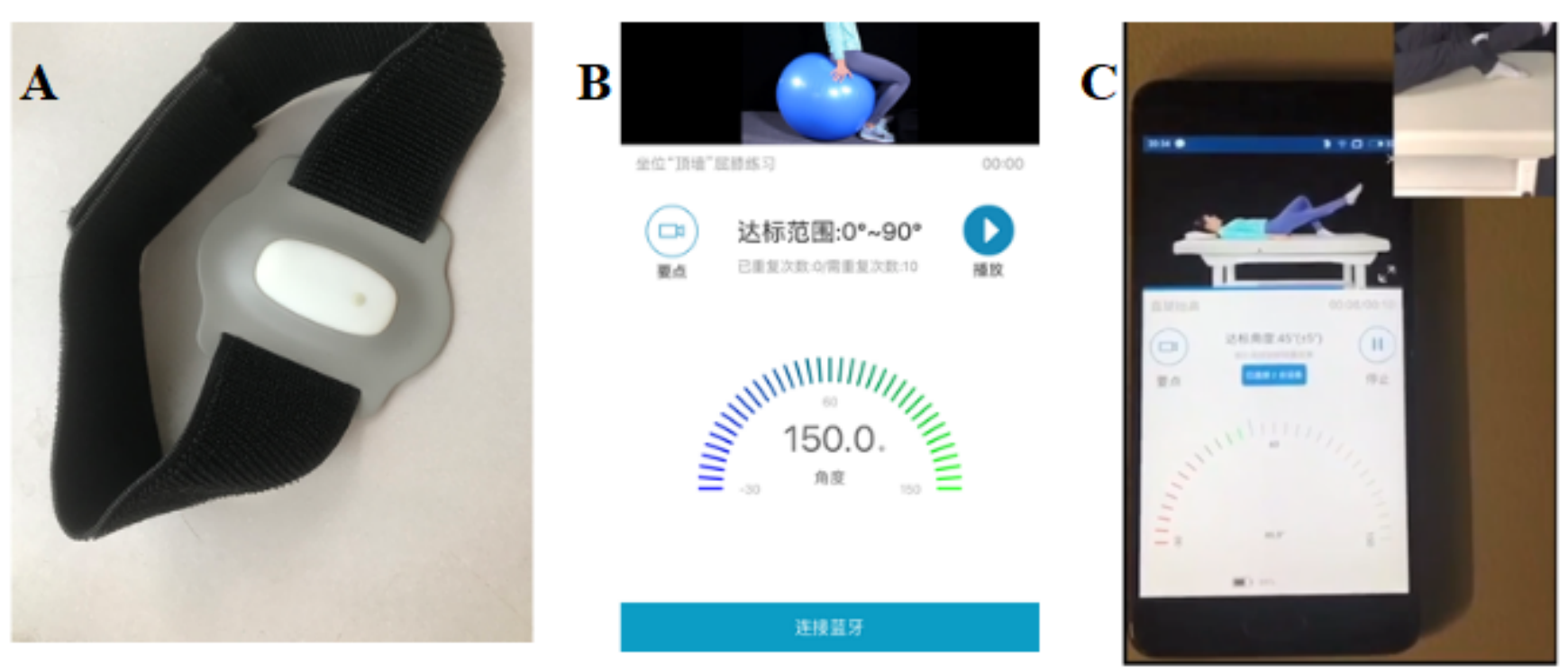

\section{Figure 2}

(A) The motion-capture device is based on six axis inertial sensor and it can monitor the patients' range of motion etc. (B) The software interface: the upper part shows the video guidance, and the lower part is the instrument panel to display the range of motion. (C) Patients rehabilitate using the system: The patient can rehabilitate according to the video guidance. Meanwhile the information such as the range of motion is transmitted back to the mobile phone by the motion-capture device they wear, and displayed on the mobile phone, so that the patients can know whether their actions reach the standard. 


\begin{tabular}{|c|c|c|c|c|c|c|}
\hline \multirow[b]{3}{*}{ Time point* } & \multicolumn{6}{|c|}{ Study Period } \\
\hline & \multirow{2}{*}{$\begin{array}{c}\text { Enrollment } \\
\text { Pre-visit }\end{array}$} & \multirow{2}{*}{$\begin{array}{l}\text { Allocation } \\
\text { Initial-visit }\end{array}$} & \multicolumn{4}{|c|}{ Postallocation } \\
\hline & & & 0 & $\begin{array}{c}3 \\
\text { months }\end{array}$ & $\begin{array}{c}6 \\
\text { months }\end{array}$ & $\begin{array}{c}12 \\
\text { months }\end{array}$ \\
\hline \multicolumn{7}{|l|}{ Enrollment: } \\
\hline Eligibility screen & $x$ & & & & & \\
\hline Informed consent & & $x$ & & & & \\
\hline Baseline assessment & & $x$ & & & & \\
\hline Allocation & & $x$ & & & & \\
\hline Surgical repair of the rotator cuff & & & $x$ & & & \\
\hline \multicolumn{7}{|l|}{ Interventions: } \\
\hline \multicolumn{7}{|l|}{ clinic and booklet rehabilitation } \\
\hline \multicolumn{7}{|l|}{ mobile phone and motion-capture devi } \\
\hline & & & & & & \\
\hline \multicolumn{7}{|l|}{ Assessments: } \\
\hline \multicolumn{7}{|l|}{ ASES } \\
\hline \multicolumn{7}{|l|}{ ROM } \\
\hline \multicolumn{7}{|l|}{ VAS } \\
\hline \multicolumn{7}{|l|}{ EQ-5D } \\
\hline \multicolumn{7}{|l|}{ UCLA } \\
\hline Retear rate & & & & & & $x$ \\
\hline Adverse events & & & & & & $x$ \\
\hline
\end{tabular}

\section{Figure 3}

Time points for enrollment, interventions and assessments

\section{Supplementary Files}

This is a list of supplementary files associated with this preprint. Click to download. 
- SPIRITchecklist.doc

Page 20/20 